\title{
The effect of COVID-19 infection on retinal nerve fiber layer and ganglion cell complex layer thicknesses
}

\author{
Sedat Ozmen', Burcin Cakir', Huseyin Dogus Okan², Nilgun Ozkan Aksoy', Ertugrul \\ Guclu $^{2}$ \\ ${ }^{I}$ Department of Ophthalmology, Sakarya University Medical Education and Research Hospital, Sakarya, Turkey \\ ${ }^{2}$ Department of Infection Diseases Sakarya University Medical Education and Researh Hospital, Sakarya, Turkey
}

\section{ABSTRACT}

Aim: To evaluate the possible effects of SARS-CoV-2 infection on retinal nerve fiber layer (RNFL) and ganglion cell complex layer (GC-IPL) thicknesses.

Method: Patients who had been infected by SARS-CoV-2 and hospitalized because of severe pneumonia were found out from the database of COVID-19 pandemic hospital and formed the patient group. The control group was composed of non-COVID-19 age-matched subjects. The mean and fragmented RNFL and GC-IPL thicknesses were measured by optical coherence tomography (OCT), and compared between two groups, statistically.

Results: Patient group 34 eyes of 34 subjects (18 male, 16 female) and 31 eyes of 31 subjects (14 male, 17 female) in the control group were enrolled. The mean age and gender were not statistically different between groups (p:0.56, 0,57, respectively).A statistically significant difference was not found between groups in terms of mean, superior, inferior, temporal, nasal RNFL thicknesses and mean superior, inferior, temporal, and nasal GC-IPL thicknesses.

Conclusion: The mean and fragmented RNFL and GC-IPL thicknesses measured by OCT were not statistically different in patients who had moderate disease course and recovered from COVID-19 infection.

Key words: COVID-19 infection, retinal nerve fiber layer thickness, ganglion cell- internal plexiform layer thickness, optical coherence tomography.

$\triangle D r$. Sedat Ozmen,

Department of Ophthalmology, Sakarya University Medical Education and Research Hospital, Sakarya, Turkey.

E-mail: drsozmen@gmail.com

Received: 2021-02-19/Revisions: 2021-03-27

Accepted: 2021-05-06 / Published online: 2021-07-01

\section{Introduction}

The novel Coronavirus disease (COVID-19) became a pandemic in the early months of 2020 [1]. We have already learned plenty of information about coronaviruses and the disease. These viruses are enveloped positivesense RNA viruses that primarily target the human respiratory system and commonly cause fever, cough, and fatigue [2,3]. Although clinical features revealed by a chest computer tomography scan present as pneumonia, the disease can also be manifested by extreme symptoms that may lead to death [4].

Ophthalmic manifestations in patients infected by a novel coronavirus (SARS-CoV-2) have been reported. Follicular conjunctivitis, conjunctival hyperemia, tender palpable 
preauricular lymph nodes, and watery discharge were found to be common ocular findings in patients with active COVID-19 [57]. To the best of our knowledge, there are few studies about the possible effects of SARSCov-2 virus infection on the posterior segment of the eye, in the literature [8-10].

The hypothesis of this current study is the effect of possible microvascular changes and neuroinflammation on inner retinal layers. The current study aimed to evaluate the possible effects of SARS-CoV-2 infection on retinal nerve fiber layer (RNFL) and ganglion cell complex layer (GC-IPL) thicknesses measured by optical coherence tomography (OCT) in patients who recovered from COVID-19.

\section{Materials and methods}

This study was conducted at Sakarya University Education and Research Hospital. Prior approval was received from the Institutional Review Board (IRB number: 71522473/050.01.04/366), and written informed consent was obtained from each subject. The study was performed in adherence to the Declaration of Helsinki.

Patients who had been infected by SARS-CoV2 and hospitalized because of severe pneumonia were found out from the database of COVID19 pandemic hospital and these patients formed the patient group. The control group was composed of patients who applied to Ophthalmology polyclinic and who had no significant ocular disease which might affect OCT assessments.

In the patient group, all patients had positive real-time reverse transcription-polymerase chain reaction (RRT-PCR) tests obtained from nasopharyngeal and oropharyngeal swabs. Oral hydroxychloroquine treatment $(200 \mathrm{mg} /$ day for 3 days) and anticoagulant therapy (enoxaparine $40 \mathrm{mg} /$ day) was given to all patients. Absence of symptoms and negative results of two consecutive nasopharangial and oropharangeal swab RRT-PCR tests were considered recovery.Two months after the recovery of COVID-19, ophthalmic examination including RNFL and GC-IPL thickness assessment was done.

Exclusion criteria for both groups were: spherical equivalent above 3 diopter, choroidal atrophy, high myopia, age-related macular degeneration, central serous chorioretinopathy, any type of glaucoma, optic neuropathy, hereditary retinal diseases, demyelinating disorders, neurodegenerative disorders, and any other ocular disorders which might alter RNFL and GC-IPL thicknesses.

Cirrus EDI-OCT (Carl Zeiss Meditec, Dublin, CA, USA) was used for peripapillary RNFL and GC-IPL thickness measurements. RNFL thickness analysis was done by "Optical Disc Cube $200 * 200 "$ method and ganglion cell analysis was done according to "Macular Cube $512 * 128$ " program.

Average, superior, inferior, temporal and nasal quadrant RNFL and GC-IPL thicknesses were compared between the patient and control groups.

Data were statistically evaluated by using IBM SPSS Statistics Software (Version 23.0). All data were reported as mean \pm standard deviation. The normality for the distribution of variables was determined by the KolmogorovSmirnov test. A student t-test was used for comparing independent samples. Any $\mathrm{p}$-value of less than 0.05 was considered to be significant.

\section{Results}

Patient group 34 eyes of 34 subjects (18 male, 16 female) and 31 eyes of 31 subjects (14male, 17 female) in the control group were enrolled. The mean ages of groups were $40.0 \pm 16.6$ years 
and $42.2 \pm 14.0$ years in the patient and the control groups, respectively. The mean age and gender were not statistically different between groups ( $P>0.56$ and $P>0.57$, respectively). Table 1 reveals characteristics of study group including additional systemic diseases, therapies, etc.

A statistically significant difference was not found between groups in terms of mean, superior, inferior, temporal, nasal RNFL thicknesses and mean superior, inferior, temporal, and nasal GC-IPL thicknesses. Table 2 reveals the details of these data.

Table 1. Characteristics of the study group.

\begin{tabular}{|l|c|}
\hline Variables & Patients (N / \%) \\
\hline Systemic arterial HT & $6 / 17$ \\
\hline DM & $2 / 5$ \\
\hline $\begin{array}{l}\text { Red eye during } \\
\text { infection }\end{array}$ & $13 / 38$ \\
\hline $\begin{array}{l}\text { Oxygen therapy } \\
\text { requirement }\end{array}$ & $18 / 52$ \\
\hline $\begin{array}{l}\text { Favipiravir } \\
\text { requirement }\end{array}$ & $12 / 35$ \\
\hline $\begin{array}{l}\text { Venous thrombosis } \\
\text { ICU admission }\end{array}$ & $4 / 11$ \\
\hline $\begin{array}{l}\text { Corticosteroid } \\
\text { requirement }\end{array}$ & $8 / 23$ \\
\hline
\end{tabular}

HT: hypertension DM: diabetes mellitus ICU: intensive care unit.

\section{Discussion}

The main possible mechanism that might affect the posterior segment of the eye in COVID-19 disease might be micro-thrombotic events in the microvascular system of the eye [8]. This type of damage to microvascular structures of the retina might cause alterations in the inner layers of the retina. Savastano et al. [8] investigated radial peripapillary capillary plexus (RPCP) perfusion density and flow index measured by OCT angiography in patients who recovered from COVID-19 disease. RPCP perfusion density was found to be lower in patients compared to controls. RNFL average thickness was linearly correlated to RPCP flow index and RPCP perfusion density within post-COVID-19 group. The RPCP perfusion density was previously found to be correlated to RNFL thickness in glaucoma patients [11,12]. The correlation between PRCP flow index and average RNFL thickness, found by Savastano et al. [8] is remarkable but they did not find statistical difference between study and control groups in terms of average RNFL and GC-IPL thicknesses. In our study, we also did not find any statistical difference. The percent of patients who required intensive care unit (ICU) administration was only $5 \%$ in our study and venous thrombosis were diagnosed in $11 \%$ of the patients in the study group. The low rate of thrombotic events might prevent microthrombotic events in the microvascular system of the eye. Patients who need ICU treatment and / or survive thrombotic events can be investigated in the future and can enlighten us on this issue. Paulo $\mathrm{M}$ et al. evaluated the retina layer of 12 patients with COVID-19 infection by using OCT in the first month of the disease and found that the patients had hyperreflective lesions in the retinal ganglion cell and inner plexiform layer, especially in the papillomacular band. In our study, we could not detect any changes in the retinal ganglion complex and retinal nerve fiber layer [13]. No retinal changes were detected in the patient group in our study. 
Table 2. Comparison of the parameters obtained from OCT between the patient and the control groups.

\begin{tabular}{|l|l|l|c|}
\hline Parameters & Patient group & Control group & p-value \\
\hline mean RNFL thickness $(\mu \mathrm{m})$ & $92,85 \pm 12,49$ & $89,58 \pm 8,40$ & 0,224 \\
\hline Superior RNFL thickness $(\mu \mathrm{m})$ & $115,70 \pm 15,87$ & $111,67 \pm 9,76$ & 0,228 \\
\hline Inferior RNFL thickness $(\mu \mathrm{m})$ & $122,79 \pm 18,18$ & $116,03 \pm 16,70$ & 0,125 \\
\hline Temporal RNFL thickness $(\mu \mathrm{m})$ & $64,94 \pm 9,68$ & $61,35 \pm 7,88$ & 0,109 \\
\hline Nasal RNFL thickness $(\mu \mathrm{m})$ & $71,85 \pm 14,68$ & $70,87 \pm 13,90$ & 0,783 \\
\hline Mean GC-IPL thickness $(\mu \mathrm{m})$ & $82,64 \pm 4,91$ & $82,22 \pm 5,38$ & 0,743 \\
\hline Superior GC-IPL thickness $(\mu \mathrm{m})$ & $82,79 \pm 6,06$ & $82,00 \pm 6,18$ & 0,603 \\
\hline Inferior GC-IPL thickness $(\mu \mathrm{m})$ & $81,44 \pm 6,06$ & $82,25 \pm 8,07$ & 0,644 \\
\hline Temporal GC-IPL thickness $(\mu \mathrm{m})$ & $82,76 \pm 4,13$ & $81,20 \pm 5,79$ & 0,239 \\
\hline Nasal GC-IPL thickness $(\mu \mathrm{m})$ & $83,02 \pm 7,68$ & $81,64 \pm 5,53$ & 0,412 \\
\hline
\end{tabular}

OCT: optical coherence tomography RNFL: retinal nerve fiber layer GC-IPL: ganglion cell-inner plexiform layer.

In their study, Burgos-Blasco et al. [14] found that patients who had COVID-19 infection with anosmia and headache during their disease period increased the thickness of the peripapillary RNFL and GC-IPL thicknesses in the early period compared to normal healthy individuals. They did not detect a difference with postcovid patients without anosmia and headache. They stated that anosmia and headache are associated with neurological involvement and that the early period of viral infection causing neuroinflammation caused an increase in RNFL and GCL [14]. In our study, we did not separate the patients into those with and without neurological findings. In the literature, there is a case report of increased intraocular pressure and angle-closure glaucoma due to change in electrolyte level after COVID-19 infection. Krawitz et al. [15] detected angle-closure glaucoma after hyponatremia due to chlortalidone use in a 65 years old patient who had positive nasopharygeal swab test for COVID-19. Hyponatremia is the most common electrolyte disorder in COVID-19 disease and has been associated with high mortality. In COVID-19 patients, serum sodium level may decrease, consequently, aqueous serum gradient changes. The higher sodium level in the humor aqueous than the serum creates an osmotic pressure difference. Consequently, fluid transition to humor aqueous occurs from serum. Fluid 
passage into humor aqueous causes increase in intraocular pressure [15]. Depending on the change in intraocular pressure, RNFL and GSIPL may also become thinner. In our study, we found no difference between the patient group and the control group in RNFL and GC-IPL. Hyponatremia was not detected in our study. The absence of RNFL and GS-IPL changes can be explained by the absence of changes in intraocular pressure.

COVID-19 patients who are treated in intensive care with acute respiratory distress are given a prone position to regulate breathing, which can increase intraocular pressure. Nerlikar et al. [16] detected bilateral angle-closure in a 56year-old diabetic and hypertensive patient who was placed in a prone position due to pneumonia due to SARS-Cov-2 infection. They stated that angle closure occurs because of the narrowing of the anterior chamber angle due to the prone position of the patient [16].

As a result, COVID 19 disease may cause changes in RNFL and GS-IPL by mechanisms such as ischemia due to microvascular occlusion in the retina and optic disc, neuroinflammation, and intraocular pressure change. Studies have shown that in COVID- 19 disease, optic nerve head RNFL and GS-IPL can be affected by different mechanisms.

There are a few studies about the effect of SARS-Cov-2 infection on inner retinal layers. In the current study, a statistically significant difference was not found in terms of RNFL and GC-IPL thicknesses between healthy controls and postcovid patients. In our opinion, more studies about the possible effect of SARS-Cov2 infection on retinal layer thicknesses should be performed.

The limitations of our study were relatively small sample sizes in both groups and alterations in the therapies. On the other hand, the severity of COVID-19 infection was moderate in all patients.

In conclusion, the mean and fragmented RNFL and GC-IPL thicknesses were not statistically different in patients who had moderate disease and recovered from COVID-19 infection.

Funding: The author(s) received no financial support for the research, authorship, and/or publication of this article.

Conflict of Interest: The authors declare that they have no conflict of interest.

Ethical statement: The study was approved by the Local Ethics Committee of Sakarya University (Decision no: 71522473/050.01.04/366), and written informed consent was obtained from each subject.

\section{Open Access Statement}

This is an open access journal which means that all content is freely available without charge to the user or his/her institution under the terms of the Creative Commons Attribution NonCommercial License (http://creativecommons.org/licenses/bync/4.0). Users are allowed to read, download, copy, distribute, print, search, or link to the full texts of the articles, without asking prior permission from the publisher or the author.

\section{References}

[1]Talevi D, Socci V, Carai M, et al. Mental health outcomes of the CoViD-19 pandemic. Riv Psichiatr. 2020;55(3):137-144.

[2]Rothan HA, Byrareddy SN. The epidemiology and pathogenesis of coronavirus disease (COVID-19) outbreak. J Autoimmun. 2020;109:102433.

[3]Wang W, Tang J, Wei F. Updated understanding of the outbreak of 2019 novel 
coronavirus (2019-nCoV) in Wuhan, China. J Med Virol. 2020;92(4):441-47.

[4]Huang C, Wang Y, Li X, et al. Clinical features of patients infected with 2019 novel coronavirus in Wuhan, China. Lancet. 2020;395(10223):497-506.

[5]Dockery DM, Rowe SG, Murphy MA, et al. The Ocular Manifestations and Transmission of COVID-19: Recommendations for Prevention. J Emerg Med. 2020;59(1):137-140.

[6]Wu P, Duan F, Luo C, et al. Characteristics of Ocular Findings of Patients With Coronavirus Disease 2019 (COVID-19) in Hubei Province, China. JAMA Ophthalmol. 2020;138(5):575-78.

[7]Öncül H, Öncül FY, Alakus MF, et al. Ocular findings in patients with coronavirus disease 2019 (COVID-19) in an outbreak hospital. J Med Virol. 2021;93(2):1126-32.

[8]Savastano A, Crincoli E, Savastano MC, et al. Against Covid-Post-Acute Care Study Group. Peripapillary Retinal Vascular Involvement in Early Post-COVID-19 Patients. J Clin Med. 2020;9(9):2895.

[9]Benito-Pascual B, Gegúndez JA, Díaz-Valle D, et al. Panuveitis and Optic Neuritis as a Possible Initial Presentation of the Novel Coronavirus Disease 2019 (COVID-19). Ocul Immunol Inflamm. 2020;28(6):922-25. [10]Cozzupoli GM, Savastano MC, Falsini B, et al. Possible Retinal Impairment Secondary to Ritonavir Use in SARS-CoV-2 Patients: A Narrative Systematic Review. J Ophthalmol. 2020;2020:5350494.

[11] Jia Y, Simonett JM, Wang J, et al. WideField OCT Angiography Investigation of the Relationship Between Radial Peripapillary Capillary Plexus Density and Nerve Fiber Layer Thickness. Invest Ophthalmol Vis Sci. 2017;58(12):5188-94.
[12] Mansoori T, Sivaswamy J, Gamalapati JS, et al. Radial Peripapillary Capillary Density Measurement Using Optical Coherence Tomography Angiography in Early Glaucoma. J Glaucoma. 2017;26(5):438-43.

[13] Marinho PM, Marcos AAA, Romano AC, et al. Retinal findings in patients with COVID19. Lancet. 2020;395(10237):1610.

[14]Burgos-Blasco B, Güemes-Villahoz N, Vidal-Villegas B, et al. Optic nerve and macular optical coherence tomography in recovered COVID-19 patients. Eur J Ophthalmol. 2021:11206721211001019.

[15] Krawitz BD, Sirinek P, Doobin D, et al. The Challenge of Managing Bilateral Acute Angle-closure Glaucoma in the Presence of Active SARS-CoV-2 Infection. J Glaucoma. 2021;30(3):e50-e53.

[16] Nerlikar RR, Palsule AC, Vadke S. Bilateral Acute Angle Closure Glaucoma after Prone Position Ventilation for COVID 19 Pneumonia. J Glaucoma. 2021. doi: 10.1097/IJG.0000000000001864. 